\title{
Germanica
}

GERMANICA $7 \mid 1990$

Grenze und Entgrenzung

\section{Grenze - Entgrenzung in Herta Müllers Prosaband:}

Der Mensch ist ein grosser Fasan auf der Welt

\section{Nicole Bary}

\section{CpenEdition}

\section{Journals}

Édition électronique

URL : http://journals.openedition.org/germanica/2502

DOI : 10.4000/germanica.2502

ISSN : 2107-0784

Éditeur

Université de Lille

Édition imprimée

Date de publication : 30 juin 1990

Pagination : 115-121

ISSN : 0984-2632

Référence électronique

Nicole Bary, «Grenze - Entgrenzung in Herta Müllers Prosaband: », Germanica [Online], 7 | 1990, Online erschienen am: 20 Mai 2014, abgerufen am 06 Oktober 2020. URL : http://journals.openedition.org/ germanica/2502 ; DOI : https://doi.org/10.4000/germanica.2502

Ce document a été généré automatiquement le 6 octobre 2020.

(C) Tous droits réservés 


\title{
Grenze - Entgrenzung in Herta Müllers Prosaband:
}

\author{
Der Mensch ist ein grosser Fasan auf der Welt
}

\author{
Nicole Bary
}

1 Sprachliche, kulturelle und politische Grenzen stecken den Rahmen ab für Herta Müllers Erzählung Der Mensch ist ein grosser Fasan auf der Welt: Grenzen zwischen eigentlichen und deutschsprachigen Rumänen, Grenzen zwischen der kleinen Zigeunerin und den Dorfbewohnern, Grenzen zwischen dem Polizisten als Vertreter der Machthabenden und den anderen. Windisch, dessen Person im Mittelpunkt der Erzählung steht, versucht, mit dem Konflikt fertig zu werden, der ihn beklemmt, seit er beschlossen hat, sein Dorf und Rumänien zu verlassen, um in die Bundesrepublik auszuwandern.

2 Herta Müller ist wie Windisch im Banat geboren, einer Gegend im Westen Rumäniens, die seit dem 18. Jahrhundert eine Enklave deutscher Sprache und Zivilisation auf rumänischem Boden geblieben ist. Die gegenwärtige politische Lage Rumäniens hat zu tragischen Zuständen geführt, an denen Menschenschicksale zerbrechen. Für die deutschsprachige Gemeinschaft in Rumänien kommt noch hinzu, dass ihr Verhältnis zu Deutschland häufig zweideutig ist, denn das Dritte Reich hat auch in Rumänien düstere Spuren hinterlassen, die die Beziehung dieser Aussiedler zu ihrem Ursprungsland deutlich belastet haben.

3 Weggehen bedeutet für Windisch: in den Westen ziehen, in ein Land, dessen Sprache er zwar spricht oder zu sprechen glaubt, über dessen Wirklichkeit, Wertmasstäbe und Bezugspunkte er aber nicht Bescheid weiss. Die Briefe, die die Dagebliebenen von den Fortgereisten bekommen, können Unsicherheit und Angstgefühle der ersten nur noch verstärken. Ob die Grundbedürfnisse im Westen, diesem Schlaraffenland, wohl befriedigt werden können? In Deutschland ist der Arbeitsmarkt für traditionnelles Handwerk, so wie man es in Rumänien noch betreibt, wie beispielsweise Glasherstellung undver arbeitung, aussichtslos. Windisch, der Dorfbewohner, Müller und Bauer, dem der ländliche Alltag vertraut ist, wandert aus in eine ver städterte, industrialisierte Welt, in der die zwischenmenschlichen Beziehungen Regeln 
unterworfen sind, die er nicht kennt, deren bedrohlichen Charakter er aber von vornherein von sich weist. «Ich würde kein Heimweh kriegen, [...] dort ist man doch nur unter Deutschen». Gleich darauf dämpft er jedoch seine Begeisterung: «Dort sind mehr Fremdnationen als hier. Dort sind Türken und Neger. Die vermehren sich rasch».

In Herta Müllers Buch verknüpfen sich reine Erzählungen und Dialoge, die von den verschiedenen Figuren geführt werden und den Bruch - die Grenze - offensichtlich machen: Bruch zwischen Ländlichkeit und Industrialisierung, zwischen traditionsbewusstem, die alten Sitten und Gebräuche respektierenden Verhalten und persönlichen Entscheidungen; zwischen Aberglauben und kritischer Geisteshaltung; zwischen dem Dorf, das Windisch verlassen und der Stadt, die er bewohnen wird. In die westliche Industriegesellschaft eintreten bedeutet, sich ihr anzupassen und, wie Windischs Frau, die Kleider- und Frisurenmode nachzuahmen, wie Tochter Amalie, ein Deodorant zu benutzen und, wie Windisch selbst, ein Auto zu fahren (er kommt im Mercedes ins Dorf zurück). Die Grenze überschreiten bedeutet für Windisch, vor allem aber für seine Frau, die Identität ablegen, die man sich bislang zu eigen gemacht hatte und sich in ein neues Wertesystem einfügen. «Als hätten wir nie hier gewohnt», sagt Windischs Frau, als sie in das Dorf zurückkehrt und die geneigten, roten Dächer betrachtet. Sie holt eine Tafel Ritter-Sport-Schokolade aus der Tasche und hält sie der Postfrau hin; die aber hatte schon ihre Wange zum Kuss hingehalten. Windischs Frau steht nunmehr jenseits der Grenze; zwischen ihr, der Fortgezo enen, und den anderen, Dagebliebenen, ist eine unsichtbare, aber undurchdringliche Barriere enstanden, die die Kommunikation zwischen den Menschen verhindert.

5 Herta Müllers Erzählung ist auch zeitlich genau abgegrenzt: sie beginnt mit dem Zeitpunkt, zu dem Windisch beschliesst, mit seiner Familie auszuwandern und endet mit dem Abreisetag. Das allerletzte Bild stellt einen geschickten Kontrapunkt zum täglichen Einerlei vor der Abfahrt dar und beschreibt einen scheinbar banalen Tag: Windisch und seine Frau haben sich gerade in der Bundesrepublik niedergelassen und besuchen nun ihr Heimatdorf. Die ganze Erzählung stützt sich auf die Empfindungen Windischs während der Wartezeit. Windischs Gefühle, Hoffnung, Bedauern, Unsicherheit, Wut bilden das Prisma, durch das der Leser den Mikrokosmos des Dorflebens entdeckt. Die Dorfbewohner sind, je nach der Funktion und Macht, die sie ausüben, genau in Kategorien eingeteilt. Die Grenze zwischen Rumänen und Deutschstämmigen teilt alles ein und jedes Individuum einer der beiden Gruppen zu, die einander Hass und Verachtung entgegenbringen. Auf das deutsche Schimpfwort «Walachen» reagieren die rumänischen Traktoristen mit einem «Nix mehr deutsch», was bedeutet, dass Rumänien den Rumänen gehört und die Deutschen sich zumindest bemühen sollten, die Landessprache zu sprechen. «Der Pfarrer sagt, dass die Gräber der Rumänen nicht zum Friedhof gehören. Dass die Gräber der Rumänen anders riechen als die Gräber der Deutschen».

6 Als Vertreter der Macht und ihrer Willkür stehen der Pfarrer und der Polizist zu der übrigen Bevölkerung in einem HerrenSklaven-Verhältnis. Beide sind sich der Tatsache bewusst, dass Windisch auf ihre Mithilfe angewiesen ist, um die notwendigen Auswanderungspapiere zu erhalten, und beide zwingen Windisch und seine Tochter dazu, ihre Bedingungen zu erfüllen. Der Vater muss mit Weizen, aber auch mit klingender Münze zahlen; die Tochter muss den Vertretern der politischen und gesellschaftlichen Ordnung zu Willen sein. Windisch ist in dem Buch die Person mit dem ausgeprägtesten Gewissen; er kann sich zwar damit abfinden, diesen zwei üblen 
Gestalten ein Schmiergeld zuzustecken, aber nicht mit dem Tugendopfer seiner Tochter. Amalie hingegen, die sowieso schon an der Härte des Lebens zerbrochen ist, geht fast wohlwollend auf die Gelüste des Pfarrers und des Milizmannes ein. Diese Szene, die ziemlich am Ende des Buches beschrieben wird, ist der Punkt, von dem aus eine Umkehr nicht mehr möglich ist; hier scheiden sich Windischs Abreisegedanken in Vorher und Nachher. Hier ist die Schwelle erreicht - die Grenze - zwischen dem, womit man noch leben konnte und dem, was man moralisch nicht mehr verkraften kann. Zeitlicher Ablauf und Rhythmus der Erzählung beschleunigen sich in den darauffolgenden Bildern. Windisch ist schon woanders, in einem Anderswo, das ihn für die Leiden der Mitmenschen, in diesem Fall seiner Tochter, empfänglich gemacht hat. «Ich weiss», sagt er, «Abschiede sind schwer. [...] Man geht und weiss nicht, ob und wie und wann man wiederkommt».

7 «Seit Windisch auswandern will, sieht er überall im Dorf das Ende. Und die stehende Zeit für die, die bleiben wollen. Und dass der Nachtwächter dableibt, sieht Windisch, über das Ende hinaus». Windisch hat sich für das Leben, für die vergehende Zeit entschieden. Er hat beschlossen, eine Zukunft zu haben, fortzugehen, die Grenze, die das Dorf, das Land, sein Leben umgibt, zu durchbrechen. Die getroffene Wahl, seine Entscheidung, zeigt auch, dass ihm dies bewusst geworden ist: Dableiben und Tod sind eins. Herta Müller lässt anklingen, dass es nur drei mögliche Antworten auf die Tragik des Lebens in diesem Banatdorf gibt: fortgehen wie Windisch, sterben wie Dietmar oder die alte Kroner oder verrückt werden wie Rudi. Drei Möglichkeiten, sich über eine Situation, die zu hart ist, als dass man damit leben könnte, hinwegzusetzen.

8 Massgebend in diesem Dorfleben ist der Tod. Andere wichtige Einschnitte im Leben, wie Geburten und Hochzeiten, die ebenfalls Anlass zu gemeinsamem Feiern gäben, spielen hier überhaupt keine Rolle. Die Eule verkörpert diese Allgegenwärtigkeit des Todes. Wie die Eule, die durch das Dorf huscht und über den Dächern kreist, scheint auch der Tod auf der Lauer zu liegen. Das Dorf hat «seine» Eule, die die Bewohner, deren Lebensgeschichten und Gewohnheiten kennt; die Eule weiss. Wenn sie sich auf dem Dach eines Hauses niederlässt und der Dorfgemeinschaft dadurch ankündigt, dass hier jemand sterben wird, dann gehorcht sie einem Gesetz, das allen Dorfbewohnern vertraut ist. Doch das Auftauchen einer jungen unerfahrenen Eule in diesem Mikrokosmos bedeutet das Eindringen von etwas Irrationalem, Unberechenbarem in die alteingesessene Ordnung des Dorfes. «Die junge Eule ist irr geworden vom Glockenläuten. Sie ist zurückgeflogen ins Land. [...] An den Wasserrausch ist sie geflogen, wo Soldaten stehn. [...] Die junge Eule zündet in den roten Hagebutten ihre Augen an. Mit den Flügeln überm Stacheldraht wünscht sie sich einen Tod». Und tatsächlich fällt der junge Dietmar, von einer verirrten Kugel getroffen. «Jetzt ist es wieder wie im Krieg», sagt der Müller. Die Gesellschaftsordnung ist zerstört; die Eule hat das Gewohnheitsrecht gebrochen und die Dorfgemeinschaft in eine chaotische, kriegsähnliche Wirklichkeit gestürzt, die Unsicherheit und Angst einflösst.

9 Für Rudi, den jungen Arbeiter aus der Glasfabrik, hat die Eule sich nicht niedergelassen. Rudi ist dem unerträglichen Alltag in eine eigene Welt entflohen, die Welt der Formen und Farben der gläsernen Dinge, die er herstellt. Durch das Dazwischenschieben des farbigen Prismas wird die Realität verformt, verfremdet, anders, eine Welt für sich, eine Welt, in der Rudi sein Anderssein ausleben kann. Dagegen versuchen seine Mitmenschen, dieses Anderssein zu verdrängen. Rudis Eltern haben sich dafür eigens 
eine Geschichte zurechtgelegt. Als die Wahrheit dennoch an den Tag kommt, wird sie wie ein Schandfleck, der sich womöglich gar vererbt, dargestellt.

All diese Themenfäden, die Herta Müller zu ihrer Erzählung verwebt, sind in einer Sprache verfasst, die ebenfalls in einem Grenzbereich angesiedelt ist. Grenzbereich zwischen realistischer und surrealistischer Wahrnehmung; zwischen den Elementen, die die Autorin aus dem wirklichen Leben herausgegriffen hat und den Bildern und Visionen, die diese Realität ihrer Phantasie diktiert. Herta Müller lockt den Leser mit ihrem Schreibstil in eine Welt, in der das Wort eine magische Kraft hat.

Dazu zwei Beispiele, die mir besonders aufschlussreich zu sein scheinen: Der Apfelbaum und Die brennende Kugel.

Die Geschichte vom Apfelbaum, der seine Äpfel selber frisst, führt eine surrealistische Komponente in die Erzählung ein, die von den Dorfbewohnern nicht verstanden werden kann. Sie steht zweifelsohne als Sinnbild für die Willkür der Macht, die die Dörfler um das bringt, wovon sie leben, nämlich den Äpfeln. Der Apfelbaum wird nun als eine Gefahr empfunden (der Dorfrichter kommt mit der Axt, die Bauern mit Mistgabeln), als eine Bedrohung, die überall Angst auslöst (im Dorf kann niemand mehr schlafen und die Hunde bellen nicht mehr), vor allem aber empfindet man das Geschehen als eine Strafe (die verzahnten Räder der Kirchenuhr sollen die Zeit der Sünde nicht messen). Die ständige Diskrepanz zwischen dem konkreten, beschreibenden Wort und der irrealen, wenn nicht gar surrealen Situation lässt nach und nach mit feinen Pinselstrichen das Abbild der Denk-und Verhaltensweisen einer Gesellschaft entstehen, die, in Aberglauben und Obskurantismus befangen, ohne weiteres manipulierbar ist.

13 In der Geschichte von der brennenden Kugel, die Windisch auf der Zunge hat, wenn er Alkohol trinkt, werden in elliptischer Erzählweise Windischs Gedankenbilder und Sinnestäuschungen sichtbar gemacht. Der Angstzustand des Müllers Windisch wächst im gleichen Masse wie sein schlechtes Gewissen. Er hat es nicht verhindern können, dass seine Tochter mit dem Pfarrer und dem Milizmann ins Bett musste, um die nötigen Papiere und Bescheinigungen für die Ausreise zu beschaffen. Die Lage ist unerträglich, sein einziger Ausweg der Alkohol. Herta Müller nennt nur die Epiphenomene des Trinkens: die knarrende Schranktür, die Hitze, die der Alkohol im Körper verbreitet, das nachfolgende Kopfweh. Der Ort, das Schlafzimmer, in dem sich diese Szene abspielt, verschwindet völlig hinter den Visionen, die im Kopf des Müllers herumspuken: der Milizmann, der in diesem Augenblick seine Tochter zum Beischlaf zwingt, das Gesicht des Polizisten, der ihn verhöhnt, wird zur Obsession. Die Vision ist zugleich Ausdruck von Windischs Scham, von der Kapitulation seines Gewissens und von seiner Unfähgkeit, diese Situation zu verkraften, obwohl er sie selbst verursacht hat.

14 Nicht nur in dieser und den beiden vorangegangenen Prosabänden Herta Müllers, sondern auch in der Erzählung Reisende auf einem Bein ist die Thematik von Grenze, Grenzüberschreitungen und Entgrenzung dominierend. Erlebnisse, Erinnerungen, Wahrnehmungen, die diese Thematik umkreisen, sind die Fäden, die die Autorin aus dem Gewebe ihrer Wirklichkeitserfahrung heraustrennt, auseinanderreisst, um sie dann in der Bildhaftigkeit ihrer Sprachrealität wieder miteinander zu verweben.

Mit den merkwürdigen Sonden der Phantasie und dem Seismographen der Sprache forscht, erkundet und entdeckt Herta Müller das unbekannte, mythische, widersprüchliche Land der Kunst. Denn es handelt sich, wie immer in der Literatur, um 
das Sehen von bisher Ungesehenem, um das Benennen des bisher Unbenannten, welches zwar nicht an sich der verändernde Zugriff auf die Welt ist, aber die Voraussetzung jeder Veränderung, jedes Überschreitens einer Grenze. 